\title{
FLORE ADVENTICE SOUS PALMERAIE, DANS LA ZONE DE SAVANE INCLUSE A DABOU, BASSE CÔTE D'IVOIRE
}

\author{
K. TRAORE' , D. SORO², C. B. PENE ${ }^{3}$ et S. AKE ${ }^{4}$ \\ 1Unité Régionale d'Enseignement Supérieur (URES) de Daloa/ Université d'Abobo-Adjamé. E-mail : kadytrao@yahoo.fr \\ 'Laboratoire de Botanique, UFR Biosciences, Université d'Abidjan-Cocody, 22 BP 582 Abidjan 22. \\ ${ }^{3}$ SUCAF-Cl, Ferkessédougou, E-mail : cbpene@yahoo.com \\ ${ }^{4}$ Laboratoire de Physiologie Végétal, UFR Biosciences, Université d'Abidjan-Cocody, 22 BP 582 Abidjan 22.
}

\begin{abstract}
RESUME
La flore adventice sous palmeraie, a été évaluée à partir d'un échantillonnage stratifié, à l'aide de 4 paramètres écologiques. Ainsi, 164 espèces d'adventices ont été recensées sur deux sites (Station expérimentale CNRA et plantations villageoises), près de Dabou, en zone de savane incluse. Une dizaine de familles botaniques se sont avérées les mieux représentées, avec 70 et 59 espèces, respectivement, en station expérimentale et en plantations villageoises. La comparaison de ces agro-écosystèmes, au moyen du coefficient de similitude, révèle une homogénéité floristique (Cs $=58 \%$ ). Parmi les principaux types biologiques recensés, les Microphanérophytes ont eu une meilleure représentativité, avec 26 et $33 \%$, respectivement, en station expérimentale et en plantations villageoises. Le cumul des autres types biologiques les plus fréquents, au sein de la flore adventice (Chaméphytes, Géophytes, Hémicryptophytes, Nanophanérophytes et Thérophytes) a donné une représentativité de $63 \%$, en station expérimentale et $58 \%$ en plantations villageoises. Quant à la loi de concentration de la flore adventice, elle a été sensiblement du type (25/75).
\end{abstract}

Mots-clés : Palmier à huile, adventice, inventaire floristique, variable écologique, savane incluse, Côte d'Ivoire.

\author{
ABSTRACT \\ WEEDS IN DABOU OIL-PALM OCHARDS IN INCLUDED-SAVANAH AREAS OF SOUTHERN IVORY COAST
}

Weed inventory was carried out at two sites in oil-palm orchard, in the Dabou, southern Côte d'Ivoire, to assess floristic diversity and risks involved as prerequisites for developing relevant weed management strategies. A stratified sampling method was used, with 4 eco-variables, as descriptors. Most represented weed species belongint to only 10 families were distributed into 70 and 59 species, in both experimental fields and farmers plantations, respectively. Among the main biological groups inventoried, Microphanerophytes were mostly represented with, 26 and $33 \%$ relative frequencies, respectively. These species were followed by Nanophanerophytes, with 28 and 20 relative frequencies, respectively. The other biological groups, as a whole, were $63 \%$ on the experimental station and $58 \%$ on farmers' plantations. The Dabou agro-ecosystems were found to be floristically homogenous, with a uniformity coefficient of $58 \%$ and a (25/75) type weed concentration law.

Key words : Oil palm, weed, floristic inventory, ecological variable, included-savannah, Ivory Coast.

\section{INTRODUCTION}

La compétition des mauvaises herbes pour l'eau, la lumière, les éléments nutritifs et l'espace de développement aux cultures a un effet négatif direct (Barralis, 1977 ; Koch et al., 1982). Elles provoquent des pertes évaluées à $9,7 \%$ de la production agricole mondiale et d'environ 10 à
$56 \%$ en Afrique (Cramer, 1967). Sur ce continent, la difficulté à maîtriser l'enherbement explique généralement les pratiques paysannes consistant à abandonner les anciennes parcelles au profit de nouvelles (Boraud, 2000). Au Tchad, il a été établi que le premier sarclage effectué à $10 \mathrm{j}$ après semis est déterminant pour la réussite de la culture et que chaque journée de retard faisait perdre $33 \mathrm{~kg}$ de coton graine 
(Marnotte, 1997). En Afrique de l'Ouest, les pertes partielles de production, dues aux adventices, se situent entre 8 et $15 \%$, pour le riz irrigué repiqué et 25 et $30 \%$ en riziculture de bas-fonds et de plateaux (Johnson, 1997). Selon Hamdoun (1977), Hamdoun et El Tigani (1977), des pertes de 63 à $88 \%$ pouvaient être enregistrées en culture d'arachide pluviale au Soudan. Des recherches visant à montrer l'influence des mauvaises herbes dans les cultures ont permis de mettre en évidence l'importance du climat, des techniques culturales, de la culture et surtout de la période d'émergence des mauvaises herbes (Vecchio et al., 1980).

La culture du palmier à huile, introduite dans les années 1920 - 1930 en Côte d'Ivoire, a très tôt bénéficié d'un vaste programme de développement agro-industriel visant à diversifier les productions agricoles en zone forestière et à assurer l'autosuffisance du pays en corps gras. Cela explique la place importante qu'occupe la culture du palmier à huile dans l'agriculture ivoirienne. En effet, le pays est le $2^{\mathrm{e}}$ producteur africain d'huile de palme, derrière le Nigeria, et le $7^{\mathrm{e}}$ mondial, avec 70000 ha de plantations agro-industrielles et 140000 ha de plantations villageoises (Anonyme 1, 2003). En outre, il existe des unités de première et de seconde transformations, aussi bien dans le secteur industriel qu'artisanal.

Malgré cet essor, la culture du palmier à huile connaît un certain nombre de contraintes agronomiques dont les adventices constituent un fléau important. Les dégâts qu'elles causent au palmier à huile sont non visibles à l'œil nu, et se situent à plusieurs niveaux. Leur gestion permet, d'une part, de limiter la compétition avec la culture et les légumineuses de couverture associées, vis-àvis de l'eau, la lumière et les éléments nutritifs et, d'autre part, de faciliter le ramassage des graines à la récolte des régimes de palme. En culture de palmier à huile, la lutte contre l'enherbement est basée sur le sarclage et l'emploie de produits chimiques (Délorme, 1979 ; Quencez et Dufour, 1982). L'utilisation répétée d'herbicides favorise la sélection d'espèces non sensibles, devenant de plus en plus dommageables à la culture (Déat et Touré, 1983).
La connaissance de la composition de la flore adventice et de son évolution, sous l'effet des facteurs environnementaux ou phytotechniques, est un préalable indispensable à l'amélioration des techniques de lutte contre elle (Barralis et Chadoeuf, 1980). Des travaux antérieurs, portant sur l'inventaire de la flore adventice en culture du palmier à huile ont été réalisés dans la zone forestière (M'boma, 2001 ; Tchoumé, 1968) en Côte d'Ivoire. La présente étude vise à caractériser, à l'aide de différents paramètres écologiques, la flore adventice, sous palmeraie, dans la région de Dabou, en zone de savane incluse, afin de proposer, à terme, des stratégies de lutte adaptées.

\section{MATERIEL ET METHODES}

\section{SITES DE L'ETUDE}

L'étude a été conduite dans les parcelles de la station expérimentale du Centre de Recherche Agronomique (CNRA) (R. Michaux) et les plantations villageoises environnantes (Figure 1). La Station couvre une superficie de 4000 ha, et est située à environ $60 \mathrm{~km}$ à l'Ouest de la ville d'Abidjan, sur l'axe Dabou-Grand-Lahou $\left(5^{\circ} 20^{\prime}\right.$ $\mathrm{N} ; 4^{\circ} 20^{\prime} \mathrm{W} ; 5-20 \mathrm{~m}$ ). La végétation originelle est une savane incluse dans la zone de forêt de la basse Côte d'Ivoire (Caliman, 1990).

Cette savane est entretenue par les feux de brousse répétés, et est d'origine probablement paléoclimatique (Leneuf et Aubert, 1956) couverte de Poaceae (Imperata cylindrica et Brachiaria sp.), avec quelques palmiers rôniers (Borassus flabelliformis) et de bosquets (Guillaumet et Adjanohoum, 1971). La station se caractérise par un climat subéquatorial à deux saisons de pluies : l'une centrée sur le mois de juin, l'autre plus réduite se situe dans les mois d'octobre et de novembre. La pluviométrie moyenne annuelle est d'environ $1700 \mathrm{~mm}$, avec une température moyenne journalière de $26^{\circ} \mathrm{C}$ et une durée d'insolation moyenne annuelle atteignant les $1500 \mathrm{~h}$.

La station est située sur une formation sableuse qui couvre une grande partie du bassin côtier de la Côte d'Ivoire (Kilian, 1931). Selon certains auteurs (Hormus, 1978 ; Roose et Cheroux, 
1966) le site se caractérise par une homogénéité pédologique. Les sols sont essen-tiellement de type sablo-argileux avec une tendance plus argileuse dans les zones de pentes accentuées.
Le phénomène dominant dans ces sols est l'entraînement des particules par lessivage accentué par la mise en culture (Roose et al., 1981).

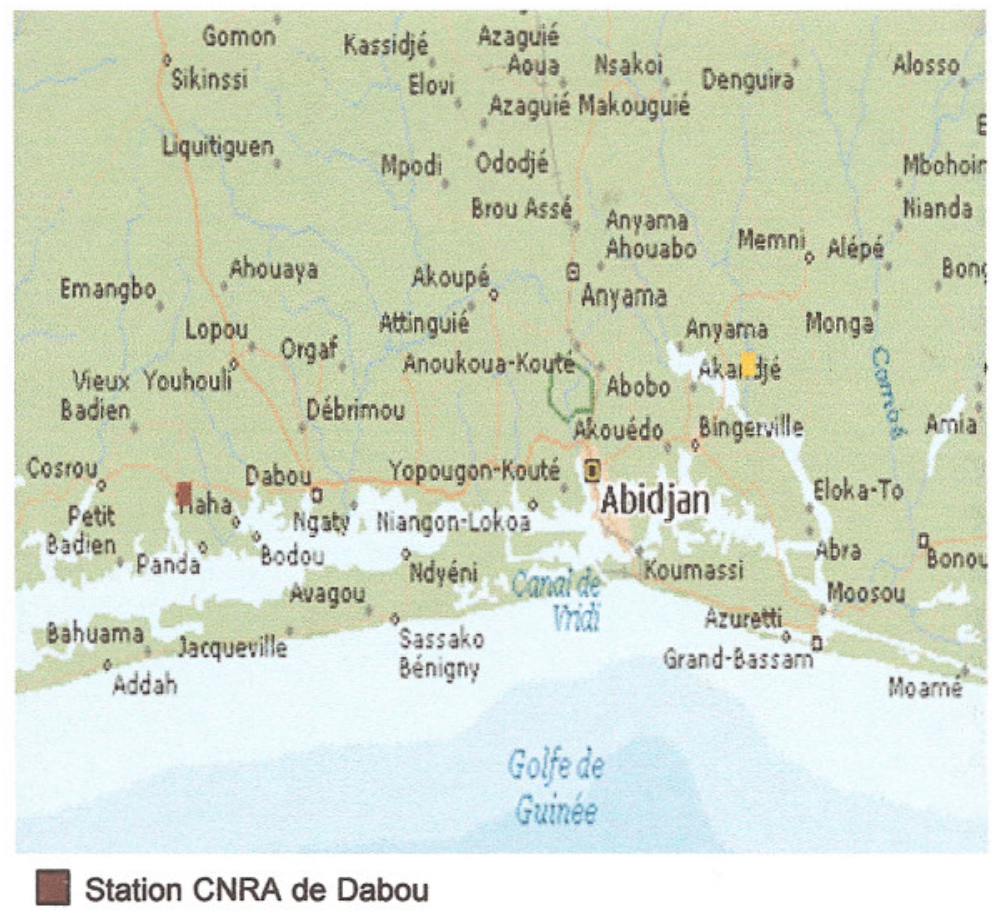

Figure 1 : Localisation du site d'étude en basse Côte d'Ivoire (Anonyme 2, 2006).

Study site location in lower Côte d'lvoire (Anonyme 2, 2006).

\section{ECHANTILLONNAGE DES ESPECES VEGETALES}

La définition d'une communauté floristique implique le choix arbitraire d'une échelle d'observation (Gounot, 1969). En culture de palmier à huile, l'unité de surface qui est préoccupante, car nécessitant un désherbage total, est le cercle autour de chaque palmier. Sur chaque site (plantations villageoises et station expérimentale CNRA), l'inventaire floristique a été effectué dans des placettes d'observation (Maillet, 1981 ; Hoffmann, 1986; Fontanel, 1987) de taille réduite à la superficie des cercles autour des palmiers $\left(7 \mathrm{~m}^{2}\right)$. Dans les interlignes et les andains, chaque placette mesure $1 \mathrm{~m}$ de largeur sur $7 \mathrm{~m}$ de longueur. L'inventaire a été réalisé suivant la méthode d'échantillonnage stratifié (Godron, 1971; Daget et Godron, 1982) avec, comme variables, 4 paramètres écologiques (Tableau 1). Chaque variable est constituée de 3 niveaux, soit 12 strates en tout. Au total, 126 et 72 relevés floristiques ont été effectués, respectivement dans les parcelles de la station Robert Michaux et dans les plantations villageoises. L'identification des espèces recensées a été faite sur place ou au Centre National de Floristique de l'Université d'Abidjan-Cocody, selon la clé dichotomique de détermination des familles (Hutchinson et Dalziel, 1954 - 1972).

Tableau 1 : Variables écologiques d'échantillonnage et les strates correspondantes.

Ecological parameters of sampling and the corresponding layers.

\begin{tabular}{|c|c|c|c|c|c|c|c|c|c|}
\hline \multirow[b]{3}{*}{ Strates } & \multicolumn{9}{|c|}{ Variables écologiques } \\
\hline & \multicolumn{3}{|c|}{ Classe d'âge } & \multicolumn{3}{|c|}{ Toposéquence } & \multicolumn{2}{|c|}{ Entité culturale } & Précédent cultural \\
\hline & Age1 & Age2 & Age3 & $\mathrm{Bf}$ & $\mathrm{Ve}$ & Pl & And & Int & $\mathrm{Sa}$ \\
\hline
\end{tabular}

Age1 : parcelles de 1 à 5 ans; Age2 : parcelles de 6 à 10 ans; Age3 : parcelles de 11 à 20 ans ; Bf : bas-fond ; Ve : versant ; $\mathrm{PI}$ : plateau ; And : endain ; Int : interligne ; Ron : rond ;Sa : savane ; Ja : jachère ; $\mathrm{Pa}$ : palmier. 


\section{Fréquence relative}

La fréquence relative $(\mathrm{Fr})$ d'une espèce végétale donnée se déduit de sa fréquence absolue ( $\mathrm{Fa}$ ) ou nombre de relevés où elle est présente et du nombre total $(\mathrm{N})$ de relevés effectués dans un certain site comme suit :

$$
\operatorname{Fr}(\%)=100 \times \mathrm{Fa} / \mathrm{N}
$$

La répartition des espèces dans chaque site (Tableau 2) a été effectuée selon l'histogramme de Raunkiaer (1905) qui comporte 5 classes de fréquence relative.

\section{Coefficient de similitude (Cs)}

II permet de vérifier l'homogénéité des sites de relevés pris deux à deux, au regard de leur composition floristique. II est déterminé selon la formule de Sorensen (1948) ci-après :

$\mathrm{Cs}=100 \times 2 \times c /(a+b)$

Où, $a$ et $b$ représentent les nombres d'espèces recensées respectivement dans les deux sites d'échantillonnage $A$ et $B$ à comparer ; c étant le nombre d'espèces communes aux deux sites A et B. II (Cs) varie de 0 à $100 \%$ selon que les deux sites sont de compositions floristiques totalement différentes $(c=0)$ ou identiques $(a=$ $b=c$ ). Pour un coefficient de similitude supérieur ou égal à $50 \%$, les deux sites concernés sont considérés comme floristiquement identiques.

\section{Contributions spécifique et floristique}

La contribution spécifique $\left(\mathrm{Cs}_{\mathrm{i}}\right)$ due à la fréquence absolue d'une espèce $i\left(\mathrm{Fa}_{\mathrm{i}}\right)$ représente son apport au sein d'une formation végétale donnée. Elle s'obtient par le rapport de la fréquence absolue de l'espèce $\left(\mathrm{Fa}_{\mathrm{i}}\right)$ à la somme des fréquences absolues de toutes les espèces rencontrées $\left({ }_{1}\right.$ $\mathrm{Fa}_{\mathrm{i}}$ ), multiplié par 100 :

$\mathrm{Cs}_{\mathrm{i}}=\mathrm{Fa}_{\mathrm{i}} / \sum_{1}^{\mathrm{n}}$ Fai $\times 100$

Elle traduit l'agressivité des espèces comme suit (Daget et Poissonnet, 1971) :
- Cs inférieure à $1 \%$ : adventice accidentelle ;

- $1 \leq \mathrm{Cs} \leq 4$ : adventices majeures potentielles ;

- Cs supérieure à 4 : adventices majeures.

A la contribution spécifique, ces mêmes auteurs ont associé une autre notion qui est la contribution floristique (Cf). Celle-ci traduit la place occupée par une espèce dans une flore donnée et s'exprime par :

$\mathrm{Cf}=(1 / \mathrm{n}) \times 100$

où, $n$ est le nombre total des espèces.

Il existe une relation du type $\mathrm{y}=\mathrm{ax}+\Delta$ (où est une autre fonction) entre $\mathrm{Cf}$ et $\mathrm{Cs}$. Le cumul des valeurs de chaque type de contribution permet de tracer la courbe de concentration. Comme $\mathrm{Cf}$ et Cs s'expriment en pourcentage, cette courbe s'inscrit dans un carré parfait et peut avoir les allures suivantes :

- si toutes les espèces rencontrées au cours de l'échantillonnage ont la même fréquence absolue (donc la même contribution spécifique), la courbe de concentration se confond à la première diagonale du carré ;

- si les espèces sont de fréquences absolues différentes, la courbe est un arc de cercle qui s'éloigne d'autant plus de la première diagonale que les espèces à fréquences absolues élevées sont en nombre réduit ;

- s'il n'existe qu'une seule espèce, la courbe se confond aux deux côtés du carré.

Selon Daget et Poissonnet (1971), la deuxième diagonale rencontre cette courbe en un point spécifique dont la somme des coordonnées est égale à 100. Ce point permet de caractériser une formation végétale donnée. Par exemple, dans une plantation d'hévéa (Aman Kadio, $1978)$, ce point a pour coordonnées $(25 / 75)$. Cela signifie que $25 \%$ des adventices de la plantation apportent une contribution spécifique de $75 \%$.

Tableau 2 : Classes de fréquences relatives des taxons selon Raunkiaer (1905).

Relative frequency classes of taxa according to Raunkiaer (1905).

\begin{tabular}{lc}
\hline Classes de fréquence & fréquence relative $(\%)$ \\
\hline Classe I & 0 à 20 \\
Classe II & 21 à 40 \\
Classe III & 41 à 60 \\
Classe IV & 61 à 80 \\
Classe V & 81 à 100 \\
\hline
\end{tabular}




\section{RESULTATS}

\section{RICHESSE FLORISTIQUE}

La richesse floristique du domaine de Dabou a été évaluée à partir de 198 relevés réalisés dans les deux sites considérés, en fonction des 4 variables écologiques étudiées (Tableaux 3 à 6 ). La flore recensée sur les deux sites comprend 164 espèces d'adventices, dont 121 espèces dans les parcelles de la station expérimentale et 111 espèces en plantations villageoises.
Parmi ces espèces, les Dicotylédones sont les plus représentées, soit $71 \%$ en station expérimentale et $75 \%$ en plantations villageoises, avec respectivement 34 et 33 familles botaniques (Tableau 7). Les Monocotylédones représentent, respectivement, $24 \%$, avec 4 familles et $21 \%$, avec 5 familles. Au sein des 10 familles les mieux représentées, 4 (Poaceae, Rubiaceae, Asteraceae, Euphorbiaceae) contiennent à elles seules $32 \%$ d'espèces dans chaque site, avec 31 genres en station expérimentale et 29 dans les plantations villageoises (Tableau 8).

Tableau 3 : Relevés d'espèces effectués dans chaque site de Dabou suivant la position dans la toposéquence.

Species counts at the Dabou sites as a function of the toposequence of the area.

\begin{tabular}{lrcrr}
\hline Sites de Dabou & \multicolumn{3}{c}{$\begin{array}{c}\text { Nombre d'espèces par } \\
\text { toposéquence }\end{array}$} & Totaux \\
\cline { 2 - 4 } & $\mathrm{Bf}$ & $\mathrm{Ve}$ & $\mathrm{Pl}$ & \\
\hline Station CNRA & 42 & 42 & 42 & 126 \\
Plantations villageoises & 24 & 23 & 25 & 72 \\
\hline Totaux & 66 & 65 & 67 & 198 \\
\hline
\end{tabular}

Tableau 4 : Relevés d'espèces effectués dans les sites de Dabou suivant la classe d'âge des palmeraies. Species counts at the Dabou sites according to palm orchard age groups.

\begin{tabular}{lcccr}
\hline \multirow{2}{*}{ Sites de Dabou } & \multicolumn{3}{c}{$\begin{array}{c}\text { Nombre d'espèces par classes } \\
\text { d'âge des palmeraies }\end{array}$} & \multirow{2}{*}{ Totaux } \\
\cline { 2 - 4 } & Age 1 & Age 2 & Age 3 & \\
\hline Station CNRA (R Michaux) & 42 & 42 & 42 & 126 \\
Plantations villageoises & 22 & 21 & 29 & 72 \\
\hline Totaux & 64 & 63 & 71 & 198 \\
\hline
\end{tabular}

Age 1 : parcelles de 1 à 5 ans; Age2 : parcelles de 6 à 10 ans ; Age 3 : parcelles de 11 à 20 ans ; $\mathrm{Bf}$ : bas-fond ; Ve : versant ; PI : plateau ; And : endain ; Int : interligne ; Ron : rond ; Sa : savane ; $\mathrm{Ja}$ : jachère ; $\mathrm{Pa}$ : palmier.

Tableau 5 : Relevés d'espèces effectués dans les sites de Dabou suivant l'entité culturale.

Species counts at the Dabou sites according to crop entity.

\begin{tabular}{lcccc}
\hline \multirow{2}{*}{ Sites de Dabou } & \multicolumn{3}{c}{ Nombre d'espèces par } & Totaux \\
\cline { 2 - 4 } & entités culturales & \\
\cline { 2 - 5 } Station CNRA (R Michaux) & 36 & Int & Ron & \\
Plantations villageoises & 20 & 26 & 45 & 126 \\
\hline Totaux & 56 & 71 & 71 & 198 \\
\hline
\end{tabular}

Age1 : parcelles de 1 à 5 ans ; Age2 : parcelles de 6 à 10 ans ; Age3 : parcelles de 11 à 20 ans ; Bf : basfond ; Ve : versant ; PI : plateau ; And : endain ; Int : interligne ; Ron : rond ;Sa : savane ; Ja : jachère ; $\mathrm{Pa}:$ palmier. 
Tableau 6 : Relevés des espèces effectués dans les sites de Dabou suivant le précédent cultural.

Species counts at the Dabou sites, as a function of the preceeding crop.

\begin{tabular}{lcccc}
\hline \multirow{2}{*}{ Sites de Dabou } & \multicolumn{3}{c}{ Nombre d'espèces par précédent cultural } & \multirow{2}{*}{ Totaux } \\
\cline { 2 - 4 } & $\mathrm{Sa}$ & $\mathrm{Ja}$ & $\mathrm{Pa}$ & \\
\hline Station CNRA (R Michaux) & 43 & 38 & 45 & 126 \\
Plantations villageoises & 31 & 22 & 19 & 72 \\
\hline Totaux & 74 & 60 & 64 & 198 \\
\hline
\end{tabular}

Tableau 7 : Répartition des effectifs d'espèces suivant les grands niveaux taxonomiques dans les sites de Dabou.

Species distribution according to great taxonomic groups at the Dabou sites.

\begin{tabular}{lcccccc}
\hline & \multicolumn{6}{c}{ Distribution par sites à Dabou } \\
\cline { 2 - 7 } Niveaux & \multicolumn{2}{c}{ Station CNRA (R Michaux) } & \multicolumn{3}{c}{ Plantations villageoises } \\
\cline { 2 - 7 } taxonomiques & Familles & Genres & Espèces & Familles & Genres & Espèces \\
\hline Dicotylédones & 34 & 70 & 86 & 33 & 70 & 84 \\
Monocotylédones & 4 & 21 & 29 & 5 & 20 & 23 \\
Ptéridophytes & 6 & 6 & 6 & 4 & 4 & 4 \\
\hline Total & 44 & 97 & 121 & 42 & 94 & 111 \\
\hline
\end{tabular}

Tableau 8 : Effectifs des familles botaniques les mieux représentées dans les sites de Dabou.

Botanical family counts, most represented at the Dabou sites.

\begin{tabular}{|c|c|c|c|c|}
\hline \multirow{3}{*}{ Familles botaniques } & \multicolumn{4}{|c|}{ Nombre de familles par sites à Dabou } \\
\hline & \multicolumn{2}{|c|}{ Station CNRA (R Michaux) } & \multicolumn{2}{|c|}{ Plantations villageoises } \\
\hline & Genres & Espèces & Genres & Espèces \\
\hline Poaceae & 14 & 17 & 12 & 17 \\
\hline Rubiaceae & 5 & 8 & 5 & 7 \\
\hline Asteraceae & 7 & 7 & 6 & 7 \\
\hline Cyperaceae & 5 & 7 & 4 & 5 \\
\hline Euphorbiaceae & 5 & 7 & 6 & 8 \\
\hline Moraceae & 4 & 7 & 2 & 3 \\
\hline Apocynaceae & 5 & 5 & 2 & 2 \\
\hline Amanranthaceae & 3 & 5 & 2 & 2 \\
\hline Fabaceae & 3 & 3 & 6 & 7 \\
\hline Verbanaceae & 2 & 4 & 2 & 4 \\
\hline Total & 53 & 70 & 47 & 59 \\
\hline
\end{tabular}

\section{FREQUENCE DES ESPECES}

La distribution des espèces, suivant I'histogramme de Raunkiaer, suit une allure unimodale en «J» inversé suggèrant que chacun des deux sites est floristiquement homogène (Figure 2). Les histogrammes se caractérisent par une forte présence d'espèces dont les fréquences relatives ont été les plus faibles (classe I) et une faible présence d'espèces à fréquences relatives élevées (classe $V$ ). Si dans le premier cas les espèces peuvent être considérées comme accidentelles, donc à nuisibilité faible, dans le second cas, l'ubiquité de Chromolaena odorata a montré une indifférence aux variations du milieu et peut donc être très recouvrante. En effet, cette espèce a constitué, avec les individus dont les fréquences relatives appartiennent aux classes III et IV, les mauvaises herbes majeures de la savane de Dabou (Tableau 9). A la station du CNRA, ces espèces, issues de 4 familles botaniques ont été : Emilia praetermissa, Nephrolepis biserrata, Heterotis rotundifolia, Oplimenus 
bumannii, Panicum laxum. Dans les plantations villageoises, les taxons concernés, issus de 6 familles ont été : Centrosema pubescens, Emilia practermissa, Nephrolepis biserrata, Oplimenus burmannii et Panicum laxum et Croton hirtus. Les espèces de la classe II ont été les mauvaises herbes majeures potentielles. Ces taxons, au nombre de 4, à la station CNRA, appartiennent à 4 familles et sont: Croton hirtus, Centrosema pubescens, Megastachya mucronata et Phymatodes scolopendria. En plantations villageoises, ils ont été issus de 6 familles : il s'agit de Albizia zygia, Anthocleista djalonensis, Clerodendrum splendens, Phymatodes scolopendri, Polygala multifolia et Spermacoce latifolia.

Les deux sites étudiés ont été floristiquement identiques, car le coefficient de similitude s'est élèvé à $58 \%$, valeur supérieure à $50 \%$, avec 67 espèces communes sur 121 dans les parcelles du CNRA et 111 en plantations villageoises. Les espèces communes se répartissent en 20 familles et 32 genres. En plus de cette homogénéité floristique, ces deux sites ont présenté une certaine similitude au niveau de la fréquence relative des espèces. En effet, sur les 10 et 14 espèces que contiennent respectivement les noyaux floristiques de base de la station CNRA et des plantations villageoises, 8 sont communes (Tableau 9).

Les principaux types biologiques rencontrés dans cette étude sont composés de Chaméphytes, Géophytes, Hémicryptophytes, Microphanérophytes, Nanophanérophytes et Thérophytes. Le tableau 10 montre l'importance relative de chacun au sein de la flore adventice du périmètre élaeicole de Dabou. Les Microphanérophytes y ont été les plus représentés, avec des fréquences relatives de 26 et $33 \%$, respectivement, dans les parcelles du CNRA et en plantations villageoises. Ils ont été suivis des Nanophanérophytes, avec, respectivement, 22 et $24 \%$. En associant le pourcentage des types biologiques (Chaméphytes, Géophytes, Hémicryptophytes, Nanophanérophytes et Thérophytes) qui ont été les plus fréquents, dans la flore adventice, on obtient $63 \%$ à la station CNRA et $58 \%$ en plantations villageoises.

\section{CONTRIBUTIONS SPECIFIQUE ET FLORISTIQUE}

Les courbes de concentration des deux sites de Dabou (station CNRA et plantations villageoises) ont été des arcs de cercle qui s'éloignent de la première diagonale (Figure 3 ). Cela explique le fait que les espèces rencontrées sur ces deux sites sont de fréquences absolues différentes et les taxons à fortes fréquences sont en nombre réduit. Par ailleurs, cette figure montre que, pour l'inventaire floristique des sites de Dabou, la relation de concentration ou d'inégalité entre les pourcentages cumulés des contributions spécifiques et floristique est respectivement de type $25 / 75$ et $26 / 74$ en station expérimentale et en plantations villageoises. Ces valeurs montrent qu'en station expérimentale, seulement $25 \%$ des espèces recensées constituent $75 \%$ de la flore adventice et qu'en plantations villageoises, $26 \%$ des taxons inventoriés contribuent pour $74 \%$ à cette flore.

Tableau 9 : Espèces d'adventices constituant le noyau floristique de base dans les sites de Dabou.

Weed species constituting the flora basis at the Dabou sites.

\begin{tabular}{|c|c|c|}
\hline \multirow{2}{*}{$\begin{array}{l}\text { Classes de fréquences } \\
\text { relatives }\end{array}$} & \multicolumn{2}{|c|}{ Espèces d'adventices dans les sites de Dabou } \\
\hline & Parcelles CNRA (RM) & Plantations villageoises \\
\hline Classe II & $\begin{array}{c}\text { C. pubescens, M. mucronata, C. hirtus, } \\
\text { P. scolopendria }\end{array}$ & $\begin{array}{l}\text { A. djalonensis, P. Multiflora, S. latifolia, } \\
\text { C. Splendens, P. scolopendria, A. zygia }\end{array}$ \\
\hline Classe III & E. praetermissa, $N$. biserrata, $P$. laxum & $\begin{array}{c}\text { C. pubescens, C. hirtus, I. involucrata, N. biserrata, } \\
\text { O. burmannii }\end{array}$ \\
\hline Classe IV & H. rotundifolia, O. burmannii & E. coccinea, $P$. laxum \\
\hline Classe V & C. odorata & C. odorata \\
\hline
\end{tabular}




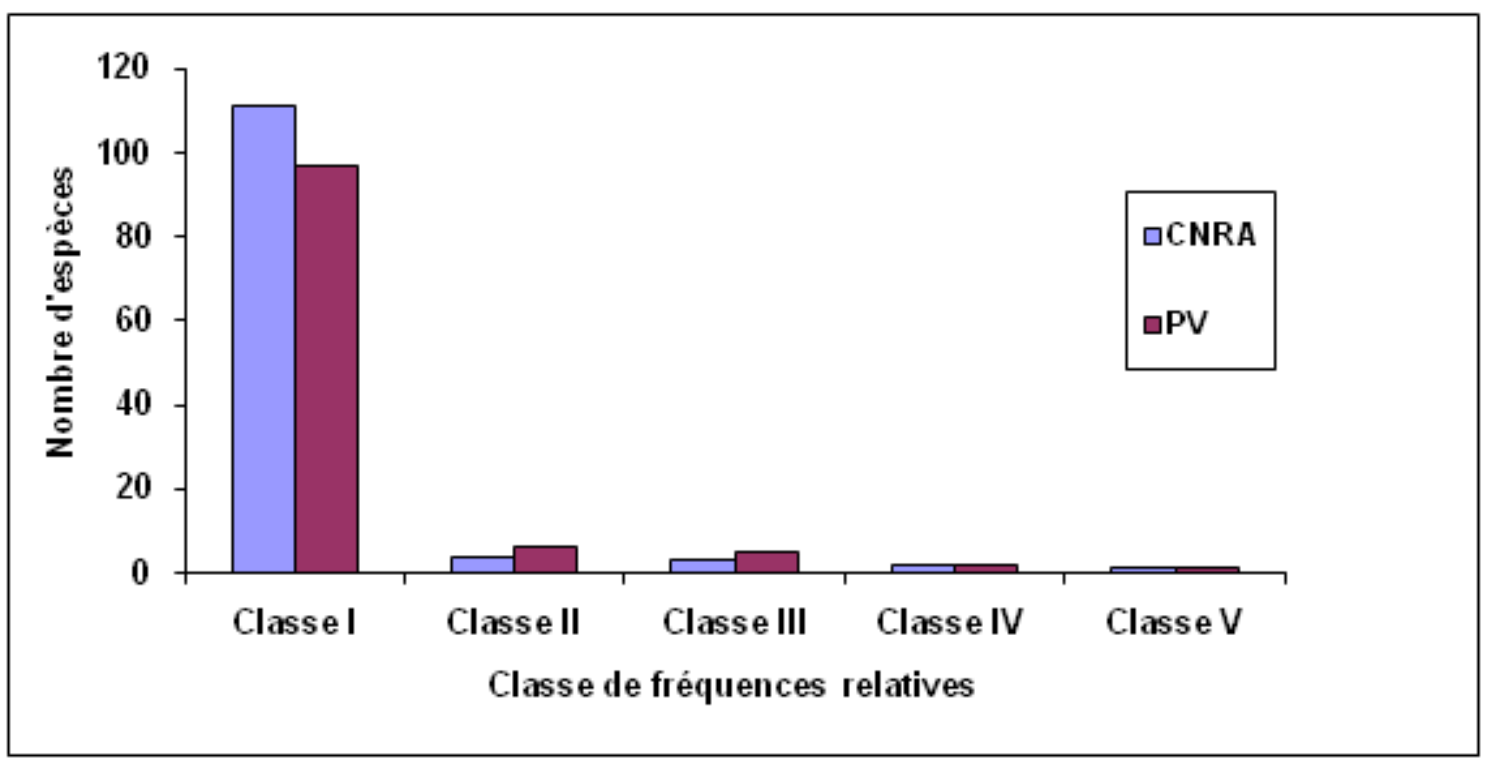

Figure 2 : Répartition des espèces végétales selon leurs fréquences relatives dans les sites de Dabou (parcelles CNRA et Plantations villageoises).

Plant species distribution according to relative frequencies at the Dabou sites.

Tableau 10 : Répartition des effectifs d'espèces selon les principaux types biologiques dans les sites de Dabou

Plant species distribution according main biotypes at the Dabou sites.

Nb espèces : nombre d'espèces ; Ch : Chaméphytes ; G : Géophytes ; H : Hémicryptophytes ; np : Nanophanérophytes ;

Th : Thérophytes ; $\mathrm{mp}$ : Microphanérophytes. Pourcentage en italique 


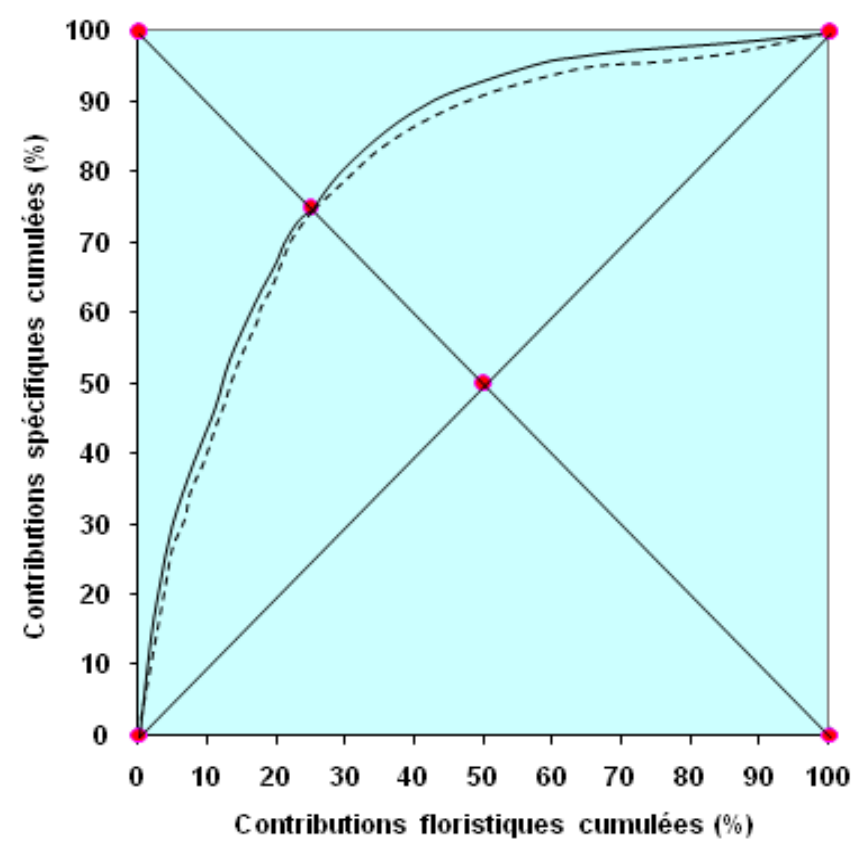

Figure 3 : Courbes de concentration des adventices en station expérimentale et en plantations villageoises dans la zone de savane incluse de Dabou, en basse Côte d'Ivoire.

Weed infestation curves at the experiment sites and under farmer conditions in the savana locked areas in Dabou, lower Côte d'lvoire.

\section{DISCUSSION}

\section{RICHESSE FLORISTIQUE}

Parmi les dix familles botaniques les plus riches en espèces à Dabou, 6 (Euphorbiaceae, Asteraceae, Poaceae, Cyperaceae, Amaranthaceae, Fabaceae) font partie des 10 familles comprenant le plus d'espèces considérées comme mauvaises herbes majeures au niveau mondial (Akobundu, 1987). II s'agit des Euphorbiaceae, Malvaceae, Asteraceae, Poaceae, Cyperaceae, Convolvulaceae, Fabaceae, Polygonaceae, Amaranthaceae et Solanaceae. Ce résultat est en accord avec ceux obtenus en Afrique de l'Ouest et du Centre (Aman Kadio et al., 2004 ; M'boma, 2001 ; Boraud, 2000 ; Le Bourgeois, 1993 ; Le Bourgeois et Guillerm, 1995 ; Traoré, 1987), relativement à la prédominance de 5 familles au sein de la flore adventice des plantes cultivées.

La répartition des espèces de Dabou, dans les proportions relatives de $2 / 3$ et $1 / 3$, respectivement, pour les Dicotylédones et Monocotylédones, est similaire aux observations faites par Boraud (2000), Le Bourgeois (1993) et Traoré (1991), au Nord de la Côte d'Ivoire et du Cameroun et au Burkina Faso. Cette répartition selon les grands niveaux taxonomiques et la hiérarchisation des familles montrent une certaine monotonie dans la diversité floristique au sein des mauvaises herbes en Afrique tropicale. En effet, en conditions pluviales, il n'est pas possible de déterminer un cortège floristique caractéristique d'une culture donnée. Seules les espèces parasites spécifiques sont inféodées à une culture, c'est le cas des espèces du genre Striga sur les céréales (sorgho, mil, maïs, riz pluvial, fonio). En climat tropical, au sein d'une unité géographique, les conditions écologiques (humidité du sol, température) sont semblables lors de la mise en place des cultures. Aucun facteur ne permet donc l'individualisation d'une flore adventice particulière à chaque culture. La localisation géographique et les pratiques culturales seront des facteurs plus discriminants que la culture (Marnotte, 2000 ; Déat, 1976).

L'abondance des microphanérophytes pourrait s'expliquer par le fait que Dabou est caractérisé par un climat subéquatorial, à pluviométrie élevée et, par l'absence de labour après plantation du palmier à huile. Les thérophytes qui sont les végétaux généralement adaptés aux milieux 
cultivés (Aman Kadio et al., 2004 ; Boraud, 2000 ; Le Bourgeois, 1993 ; Traoré, 1991 ; Hoffmann, 1986) ne représentent que $21 \%$ à la station CNRA et $14,4 \%$ en plantations villageoises. Contrairement aux microphanérophytes, ces végétaux sont héliophiles pour la plupart (Aman Kadio, 1978) et caractéristiques des milieux arides.

\section{RELATIONS ENTRE FREQUENCE, RECOUVREMENTETABONDANCE DES ESPECES}

Malgré les faibles fréquences relative dans les parcelles de palmier à huile, deux taxons (Brachiaria ruziziensis et Imperata cylindrica) sont très recouvrants lorsqu'ils sont présents. Cela s'explique par le fait que $B$. ruziziensis, une espèce introduite comme plante fourragère, soit devenue localement abondante en l'absence de bétail. L'éradication de I. cylindrica, une espèce caractéristique de la savane de Dabou, qui comporte plusieurs étapes, notamment la destruction des rhizomes, est un préalable à la mise en place des palmeraies. Lorsque cette opération est biaisée pour une raison quelconque, la plante envahit le sol et devient nuisible.

Outre ces deux espèces, certains taxons du noyau floristique de base du périmètre de Dabou, tels que Chromolaena odorata, Heterotis rotundifolia, Oplismenus burmanii s'avèrent très recouvrants dans beaucoup de parcelles. Cela est en accord avec les travaux de Marnotte (1989) et de Traoré (1991) au Burkina Faso, qui ont montré que les espèces les plus fréquentes sont les plus recouvrantes.

\section{CONTRIBUTIONS SPECIFIQUE ET FLORISTIQUE}

Les études théoriques de Jacquard et al. (1968) et de Poissonnet (1968), ont révélé une relation entre le pourcentage des espèces de la flore d'un site et les contributions spécifiques. Cette relation est assimilable à la loi de concentration, ou loi de Gini-Lorenz de type 20/80. A Dabou, la loi de concentration de la flore adventice est du type $25 / 75$, en station et $26 / 74$ en plantations villageoises. Ces valeurs sont comprises entre celles déterminées par les auteurs précités (20/ 80 ) et celles de l'étude de Daget et Poissonnet (1969) dans les prairies (23/77). De plus, ce résultat est similaire à celui obtenu par Aman Kadio (1978) dans les plantations d'hévéa (25/ 75) à l'Anguédédou, près d'Abidjan, en basse Côte d'Ivoire.

\section{CONCLUSION}

L'inventaire floristique effectué dans le périmètre de palmier à huile de Dabou a permis de recenser 164 espèces, dont 121 dans les parcelles du CNRA et 111 en plantations villageoises. Ces deux sites offrent une grande diversité floristique, avec $80 \%$ de genres différents. Les Dicotylédones y sont les plus représentées, soit $71 \%$ en station expérimentale et $75 \%$ en plantations villageoises, avec, respectivement 34 et 33 familles botaniques. Les Monocotylédones représentent, respectivement $24 \%$ et $21 \%$. Les dix familles les mieux représentées comprennent, à elles seules, 70 espèces réparties en 53 genres, en station expérimentale et 59 espèces appartenant à 47 genres, en plantations villageoises. Les types biologiques dominants de la flore sont les Microphanérophytes, avec $26 \%$ en station expérimentale et $33 \%$ en plantations villageoises. Les autres types les plus fréquents au sein de la flore adventice totalisent, respectivement, 63 et 58 en station et en plantations villageoises.

Une analyse de la flore des deux sites de Dabou laisse apparaître une homogénéité floristique avec un coefficient de similitude égal à $58 \%$. Quant à la loi de concentration de la flore adventice, elle est sensiblement du type (25/75).

\section{REFERENCES}

Akobundu I. O. 1987. Weed science in the tropics. Principles and practices. Chichester J., Wiley \& Sons Eds, 522 p.

Aman Kadio G. 1978. Flore et végétation des adventices dans l'hévéaculture en basse Côte d'Ivoire (Station expérimentale de I'I.R.C.A.). Etude écologique: Dynamique et structure. Thèse de Doctorat de spécialité, Malherbologie, FAST, Univ. d'AbidjanCocody (Côte d'Ivoire), 194 p.

Aman Kadio G., Ipou I. J. et Y. Touré. 2004. La flore des adventices des cultures cotonnières de la région du Worodougou, au Nord-ouest de la Côte d'Ivoire. Agron. Afr. 16 (1) : 1 - 14

Anonyme 1. 2003. Palmier à huile. In : Programmes de $2^{\mathrm{e}}$ Génération, Commission Cultures d'exportation. Document de travail. Abidjan, CNRA : 40 - 49.

Anonyme 2. 2006. Encarta : Microsoft corporation. C : 1993-2005. 
Barralis G. 1977. Seuils de nuisibilité des mauvaises herbes. Nuisibilité directe. Phytoma : 11 - 15.

Barralis G. et R. Chadoeuf. 1980. Etude de la dynamique d'une communauté adventice : Evolution de la flore adventice au cours du cycle végétatif d'une culture. Weed Research, $20: 231$ - 282.

Boraud N. K. M. 2000. Etude floristique et phytoécologique des adventices des complexes sucriers de Ferké 1 et 2, de Borotou-Koro et de Zuénoula, en Côte d'Ivoire. Thèse de Doctorat $3^{e}$ Cycle, Ecologie Végétale, UFR Biosciences, Univ. d'Abidjan-Cocody (Côte d'Ivoire), 157 p. + annexes.

Caliman J. P. 1990. Dégradation de propriété physiques conditionnant la fertilité des sols sous culture du palmier à huile en Côte d'Ivoire : Essai de correction. Thèse de Doctorat ès Sciences, Sciences du sol, Univ. de Bourgogne, Dijon (France), 219 p.

Cramer H. H. 1967. Plant protection and world crop production. Pflanzenschutz Nachrichten Bayer 20 : 1 - 524.

Daget P. et J. Poisonnet. 1969. Analyse phytosociologique des prairies. Applications agronomiques. Doc. 48. Montpellier, CNRS/CEPE, $67 \mathrm{p}$.

Daget P. et J. Poisonnet. 1971. Les variables écologiques et leur relevé. Versailles, $\mathrm{ENSH}, 7 \mathrm{p}$.

Daget P. et M. Godron. 1982. Analyse de l'écologie des espèces dans les communautés. Collection d'écologie. Paris, Masson, $163 \mathrm{p}$.

Déat M. 1976. Les adventices des cultures cotonnières en Côte d'Ivoire. Cot. Fib. Trop. 31 (4) : 419 - 427.

Déat M. et Y. Touré. 1983. L'expérimentation d'herbicide en culture cotonnière en Côte d'Ivoire : Résultat récents. C.R. VI e Confér. Bienn. SOAM/WAWSS. Abidjan (Côte d'Ivoire) : 236 - 247.

Délorme M. 1979. Désherbage des oléagineux pérennes : Cas particuliers des jeunes cultures et de la lutte contre les adventices nuisibles. Oléagin. 34 (1) : 1 - 4.

Fontanel P. 1987. Groupements adventices et facteurs écologiques et culturaux : comportement des espèces dans le cycle cultural, efficacité des désherbages paysans et voies d'amélioration au Sine Saloum (Sénégal). Rapport DSV 87 (7). Montpellier, CIRAD/IRAT, $60 \mathrm{p}$.
Godron M. 1971. Essai sur une approche probabiliste de l'écologie des végétaux. Thèse de Doctorat, Biologie et Ecologie Végétales, USTL, Montpellier (France), $247 \mathrm{p}$.

Gounot M. 1969. Les méthodes d'étude quantitative de la végétation. Paris, Masson et Cie Eds., 314 p.

Guillaumet J. L. et E. Adjanohoun. 1971. La végétation de la Côte d'Ivoire. In : Milieu Naturel de la Côte d'Ivoire. Mém. ORSTOM $n^{\circ}$ 50. Paris, ORSTOM : 157 - 263.

Hamdoun A. M. 1977. Competitive effects of weeds upon growth and yield of cotton, groundnuts and sorghum in the Kenana Area of the Sudan. Z. Pflanzenkrankheiten und Pflanzenschuts $84: 375$ - 405 .

Hamdoun A.M. et K. A. El Tigani. 1977. Weed control problems in the Sudan. PANS 23 (2) : $190-194$.

Hoffmann G. 1986. Caractérisation de la flore adventice de deux villages du terroir de Katiola (Côte d'Ivoire). Mémoire de fin d'études pour l'obtention du diplôme d'Ingénieur en Agronomie Tropicale, Génie Agronomique, ESAT-CNEARC Montpellier (France), $51 \mathrm{p}$.

Hormus P. 1978. Ébauche de la carte pédologique de la plantation R. Michaux. Rapport Interne. Dabou (Côte d'Ivoire), IRHO, 15 p.

Hutchinson J. et J. M. Dalziel. 1954 - 1972. Flora of west tropical Africa. London, Keay R.W.J. and Hepper F.N. (Eds), vol. 3, 828 p.

Jacquard P. Daget P., Poissonnet J. et Laroche G. 1968. Expression de l'évolution du potentiel de production et la composition botanique d'une formation herbacée dense. Doc. 47. Montpellier, CNRS/CEPE, $22 \mathrm{p}$.

Johnson D.E. 1997. Les adventices en riziculture en Afrique de l'Ouest. Bouaké, ADRAO/ WARDA, $312 \mathrm{p}$.

Kilian C. 1931. Des principaux complexes continentaux du Sahara. In : C.R. sommaire de la Soc. Géolog. de France : Paris, SGF : $109-110$.

Koch W., Beshir M. E. and R. Unterladstatter. 1982. Crop losses due to weeds. Improving weed management. FAO, Plant Prod. \& Prot. Paper 44 : 135 - 165.

Le Bourgeois T. 1993. Les mauvaises herbes dans la rotation cotonnière au NordCameroun (Afrique centrale). Thèse de Doctorat, Biologie et Ecologie Végétales, USTL, Montpellier (France), 249 p. 
Le Bourgeois T. et J. L. Guillerm J. L. 1995. étendue de distribution et degrés d'infestation des adventices dans la rotation cotonnière du Nord-Cameroun. Weed Res. 35 : 89 - 98.

Leneuf N. et G. Aubert. 1956. Pédologie sur l'origine des savanes de la basse Côte d'Ivoire. C.R. Acad. Sci. Paris 243 : $859-860$.

Maillet J. 1981. Evolution de la flore adventice dans le Montpelliérain sous la pression des techniques culturales. Thèse de DocteurIngénieur, Biologie et Ecologie Végétales, USTL, Montpellier (France), 200 p.

Marnotte P. 1989. Plantes nuisibles, désherbage, herbicides au complexe sucrier de la SOSUCO. Rapport de mission en Malherbologie à Banfora (Burkina Faso). Montpellier, CIRAD-CA, $23 \mathrm{p}$.

Marnotte P. 1997. Désherbage des cultures tropicales au COLUMA. La défense des végétaux. Phytoma $491: 59$ - 60 .

Marnotte P. 2000. La gestion de l'enherbement et l'emploie des herbicides dans les systèmes de culture en zone Soudano sahélienne en Afrique de l'Ouest et du Centre. Formation CIRAD. Montpellier, CIRAD-CA, $66 \mathrm{p}$.

M'boma R. 2001. Inventaire des adventices et étude Monographique de quelques taxon des plantations de palmier à huile (Elaeis guineensis Jacq.) de la Palmafrique, dans la forêt classée de l'Anguédédou (Côte d'Ivoire). Mémoire de D.E.A., Ecologie Végétale, UFR Biosciences, Univ. d'Abidjan-Cocody (Côte d'Ivoire), 75 p. + annexes.

Poisonnet J. 1968. Recherche sur une Ioi d'équilibre dans la composition floristique des formations herbacées denses. Montpellier, CNRS-CEPE, $18 \mathrm{p}$.

Quencez P. et F. Dufour. 1982. La lutte chimique contre les mauvaises herbes en palmeraie : Les matières actives herbicides usuelles en élaeiculture et les techniques des traitements. Oléagin. 37 (3) : $107-111$
Raunkiaer S. 1905. On biologiske typer, med hensyn til planteres tilpasning til at overleve ugunstige Aarstider, Botanisk Tidsskrift : XXVI, $540 \mathrm{p}$.

Roose E. J. et M. Cheroux. 1966. Les sols du bassin sédimentaire de la Côte d'Ivoire. Cahiers ORSTOM 4 (2) : 51 - 92.

Roose E. J., Fauck R., Lelong F., G. Pédro. 1981. Pédologie- Modifications fondamentales de la dynamique actuelle de sols ferrallitiques et ferrugineux d'Afrique occidentale sous l'influence de la mise en culture. C.R. Acad. Sci. Paris $292: 1457$ - 1460.

Sorensen T. 1948. A method of establishing group of equal amplitude in plants sociology based on similary of species content. Det Kongelige danske videnkarbernes. Biologiske Skrifter 5 (4) : 1 - 34

Tchoumé M. 1968. Etude de la végétation adventice des palmeraies à Elaeis guineensis Jacq en Côte d'Ivoire forestière. Mémoire de D.E.S., Ecologie Végétale, Univ. d'AbidjanCocody (Côte d'Ivoire), $93 \mathrm{p}$.

Traoré H. 1987. Influence des techniques culturales sur le développement des mauvaises herbes en station et en milieu rural de Côte d'Ivoire. Mémoire de D.E.A., Biologie et Ecologie Végétales, USTL, Montpellier (France), $50 \mathrm{p}$.

Traoré H. 1991. Influence des facteurs agroécologiques sur la constitution des communautés adventices des cultures céréalières (sorgho, mil, maïs) du Burkina Faso. Thèse de Doctorat, Biologie et Ecologie Végétales, USTL, Montpellier II (France), 180 p. et annexes.

Vecchio V., Vazzana C. et G. Crosta. 1980. Relations entre plantes adventices à différents niveaux d'infestation et le développement et rendement du blé. In : Actes du Vle Colloque International sur l'Ecologie, la Biologie et la Systématique des mauvaises herbes (Tome 2), Montpellier, 7 - 8 mai 1980. Montpellier, COLUMA/ EWRS : 421 - 433. 